\title{
Scale-Free Properties of the Functional Magnetic Resonance Imaging Signal during Rest and Task
}

\author{
Biyu J. He \\ National Institute of Neurological Disorders and Stroke, National Institutes of Health, Bethesda, Maryland 20892
}

It has been shown recently that a significant portion of brain electrical field potentials consists of scale-free dynamics. These scale-free brain dynamics contain complex spatiotemporal structures and are modulated by task performance. Here we show that the fMRI signal recorded from the human brain is also scale free; its power-law exponent differentiates between brain networks and correlates with fMRI signal variance and brain glucose metabolism. Importantly, in parallel to brain electrical field potentials, the variance and power-law exponent of the fMRI signal decrease during task activation, suggesting that the signal contains more long-range memory during rest and conversely is more efficient at online information processing during task. Remarkably, similar changes also occurred in task-deactivated brain regions, revealing the presence of an optimal dynamic range in the fMRI signal. The scale-free properties of the fMRI signal and brain electrical field potentials bespeak their respective stationarity and nonstationarity. This suggests that neurovascular coupling mechanism is likely to contain a transformation from nonstationarity to stationarity. In summary, our results demonstrate the functional relevance of scale-free properties of the fMRI signal and impose constraints on future models of neurovascular coupling.

\section{Introduction}

Research using fMRI has tremendously deepened our understanding of functional localizations in the brain and interactions between brain regions and networks. The overwhelming majority of fMRI studies use approaches that fall into two categories: (1) comparing the fMRI signal amplitude between behavioral conditions and deducing the functional properties of a brain region using either univariate (Posner and Raichle, 1997; Rosen et al., 1998) or multivariate (Haxby et al., 2001; Haynes and Rees, 2006; Pereira et al., 2009) statistical methods, and (2) studying the interactions between spontaneous or task-evoked fMRI signal amplitude fluctuations from different brain regions using a variety of methods, including linear correlation, Granger causality, and statistical modeling (Fox and Raichle, 2007; Friston, 2009).

Several recent studies suggested that the variance of the fMRI signal provides additional, primarily orthogonal information to the above mean-based measurements. The fMRI signal variance not only decreases in the visual cortex during task-induced activation (Bianciardi et al., 2009) but also decreases in the defaultmode network during task-induced deactivation (Fransson, 2006). Furthermore, it was shown recently that aging correlates with decreased fMRI signal variance (Garrett et al., 2010, 2011).

\footnotetext{
Received April 27, 2011; revised July 12, 2011; accepted Aug. 3, 2011.

Author contributions: B.J.H. designed research; B.J.H. performed research; B.J.H. contributed unpublished reagents/analytic tools; B.J.H. analyzed data; B.J.H. wrote the paper.

This research was supported by the Intramural Research Program of the NIH/NINDS. I am grateful to Marc Raichle for sharing the data used in this study, past teachings of brain metabolism, and comments on a previous draft of this manuscript and to Patrice Abry and Philippe Ciuciu for sharing methods used in synthesizing fractional Gaussian noise.

The author declares no competing financial interests.

Correspondence should be addressed to Biyu J. He, National Institute of Neurological Disorders and Stroke, National Institutes of Health, Bethesda, MD 20892. E-mail: biyu.jade.he@gmail.com.

DOI:10.1523/JNEUROSCI.2111-11.2011

Copyright $\odot 2011$ the authors $\quad 0270-6474 / 11 / 3113786-10 \$ 15.00 / 0$
}

Along a separate line of research, it has been known for more than a decade that the fMRI signal demonstrates temporal dependence and a power spectrum that is $1 / f$ like: $P \propto 1 / f^{\beta}$, where $P$ is power, $f$ is frequency, and $\beta$ is called the "power-law exponent" (Bullmore et al., 2001). However, there has been much reservation about the relevance of this phenomenon to brain function, primarily for two reasons. First, it is feared that instrument noise may produce artifactual $1 / f$-like signals (Zarahn et al., 1997). Second, it is sometimes argued that, because a $P \propto 1 / f^{\beta}$ power spectrum is exhibited by many natural, physical, and biological processes (Schroeder, 1991; Bak, 1996; Mandelbrot, 1999; Goldberger et al., 2002), it is unsurprising and unlikely to be important for brain function.

There is by now overwhelming evidence against the above two sentiments. First, when instrumental noise is carefully controlled for, it was found using both $\mathrm{AMRI}$ and electrophysiology that the brain does indeed produce $1 / f^{\beta}$ power spectra on its own (He et al., 2010). These signals have been termed scale-free brain activity because a $P \propto 1 / f^{\beta}$ power spectrum is indicative of scale invariance (He et al., 2010). Second, scale-free properties of the fMRI signal vary among gray matter, white matter, and CSF (Bullmore et al., 2004; Ciuciu et al., 2011) and between brain networks ( $\mathrm{He}$ et al., 2010), arguing strongly against an instrumental-noise origin. Most importantly, recent evidence showing that the powerlaw exponent of brain field potentials decreases during task activation (He et al., 2010) suggests that scale-free brain activity is functionally significant.

In this study, we rigorously tested the hypothesis that the fMRI signal is scale free and investigated its scaling properties in relation to fMRI signal variance, brain networks, metabolism, and task performance. Our results confirm the above hypothesis, demonstrate that both the dynamic range and the temporal memory of the fMRI signal decrease during task 
state, and reveal stationarity in the fMRI signal not matched by its electrical counterpart.

\section{Materials and Methods}

fMRI data acquisition. Blood oxygen-level dependent $\mathrm{fMRI}$ data $(4 \times 4 \times$ $4 \mathrm{~mm}$ voxels; TE, $25 \mathrm{~ms}$; TR, $2.16 \mathrm{~s}$ ) were acquired in 17 normal righthanded young adults using a 3 T Siemens Allegra MR scanner. Subjects were recruited from the Washington University area (nine females and eight males; age, 18-27 years). All subjects completed eight fMRI runs, each 194 frames ( $7 \mathrm{~min}$ ) in duration. They consisted of two alternating run types. The first run type was a resting-state fixation run in which a white crosshair was presented in the center of a black screen. Subjects were instructed to look at the crosshair, remain still, and to not fall asleep. The second run type was a button-press run in which the identical crosshair was presented, but now it occasionally changed from white to dark gray for a period of $250 \mathrm{~ms}$. Subjects were instructed to press a button with their right index finger as quickly as possible when they saw the crosshair dim. They were told that their reaction times would be recorded. Each of these button-press runs contained 20 crosshair dims time locked to the scanner TR, with an intertrial interval of 17.3-30.2 s. Subjects practiced this button-press task once in the scanner, before the onset of the functional scans. Anatomical MRI included a highresolution $(1 \times 1 \times 1.25 \mathrm{~mm})$ sagittal, T1-weighted MP-RAGE (TR, $2.1 \mathrm{~s}$; TE, $3.93 \mathrm{~ms}$; flip angle, $7^{\circ}$ ) and a T2-weighted fast spin echo scan.

$f M R I$ data preprocessing. fMRI preprocessing steps included the following: first, compensation of systematic, slice-dependent time shifts; second, elimination of systematic odd-even slice intensity difference attributable to interleaved acquisition; third, rigid body correction for interframe head motion within and across runs; and fourth, intensity scaling to yield a whole-brain mode value of 1000 (with a single scaling factor for all voxels). Atlas registration was achieved by computing affine transforms connecting the fMRI run first frame (averaged over all runs after cross-run realignment) with the T2- and T1-weighted structural images (Ojemann et al., 1997). Our atlas representative template included MP-RAGE data from 12 normal individuals and was made to conform to the 1988 Talairach atlas (Talairach and Tournoux, 1988). The first four frames of each fMRI run were discarded in all additional analyses. For each voxel, the time course from each fMRI run was made zero mean, and head motion and its temporal derivative were removed by linear regression.

PET data acquisition. The PET dataset used in this study was published previously by Vaishnavi et al. (2010); the methods for acquiring the data and calculating regional variations in cerebral metabolic rate for oxygen and glucose (CMRGlu), cerebral blood flow, cerebral blood volume, and glycolytic index (GI) were described in detail in that study. The method for obtaining the oxygen extraction fraction (OEF) values was described in detail previously by Raichle et al. (2001).

Definition of regions of interest. Thirty-one regions of interest (ROIs) were obtained from our previous task-related functional neuroimaging studies or generated using coordinates from published fMRI studies, which included 10 pairs of homologous brain regions. These ROIs were the same as used in our previous study (He et al., 2010, their Fig. 5). Their locations in the brain are shown in Figure 1 (mapping to brain surface was done in CARET, http://brainvis.wustl.edu/wiki/index.php/Caret: About). The regions were grouped into five cortical networks based on their known anatomical/functional properties (including attention, default-mode, motor, saliency, and visual networks) and a separate group outside the neocortex (including hippocampus, thalamus, and cerebellum).

The anatomical locations, Talairach coordinates, references, and associated networks of these ROIs are listed in Table 1. Specifically, the attention, motor, visual, thalamus, and cerebellum regions were obtained from functional studies conducted by He et al. (2007). The default network regions were obtained from task-deactivation patterns from a meta-analysis of nine PET studies, which originally unveiled the default network (Shulman et al., 1997). To generate these ROIs, following methods described by He et al. (2007), the activation or deactivation $Z$-score maps were subjected to an automatic peak search, peaks closer than 10 $\mathrm{mm}$ were consolidated by averaging their coordinates, and ROIs were defined around peaks by thresholding the map to yield regions of $\sim 905$ $\mathrm{mm}^{3}$, a similar size as the coordinates-derived ROIs described below.

The dorsolateral prefrontal cortex (DLPFC), part of the frontoparietal attentional network, and the saliency (also called "core task control") network regions were obtained from published coordinates in three studies (Dosenbach et al., 2006; Seeley et al., 2007; Vincent et al., 2008). The coordinates for Broca's area and the hippocampal formation (HF) were obtained from Embick and Poeppel (2006) and Vincent et al. (2006), respectively. In cases in which coordinates from multiple studies were obtained for one ROI, such as the right DLPFC and right temporoparietal junction (Table 1), the center-of-mass of these coordinates were used. A 6-mm-radius sphere ROI centered at these coordinates was created for each region. All regions used in the present study have been investigated in seed-based functional connectivity analyses applied to resting-state fMRI data by the author and, for ROIs in the attention and saliency networks as well as the HF, also in previous published studies (Dosenbach et al., 2007; He et al., 2007; Seeley et al., 2007; Vincent et al., 2006,2008 ) and have yielded networks consistent with those reported in the literature (Biswal et al., 1995; Fox et al., 2005, 2006; Damoiseaux et al., 2006).

In addition, following Fox et al. (2007), the left somatomotor cortex (LMC) was defined for each subject using task activation patterns from the button-press fMRI runs, and the right somatomotor cortex (RMC) was defined for each subject by using functional connectivity applied to resting-state fMRI runs and the individual subject's LMC region as seed ROI.

Power spectrum calculation. The fMRI signal time course from each ROI was extracted for each subject and fMRI run. The normalized or non-normalized power spectrum of the fMRI signal was computed using the Bartlett smoothing procedure of deriving the power spectral function from the lagged autocorrelation or auto-covariance function, respectively (Jenkins and Watts, 1998). A Tukey window of 20 fMRI frame width was applied for additional smoothing. The power spectra were then averaged across runs and subjects and across homologous ROIs, resulting in an average power spectrum for each of 21 brain regions (Fig. $2 A$ ). Finally, to obtain the power-law exponent $\beta$, the $<0.1 \mathrm{~Hz}$ range of each average power spectrum was fit with a power-law function: $P(f) \propto$ $1 / f^{\beta}$ using a least-squares fit. Using the low-frequency range to fit the power-law exponent avoids aliasing artifact in higher-frequency range (we used TR of $2.16 \mathrm{~s}$, hence Nyquist limit is $0.23 \mathrm{~Hz}$ ) and yields reliable measurement of the scale-free distribution (Eke et al., 2002).

Scale-invariance definition. Power-law scaling behavior is indicative of scale invariance: if $P(f) \propto 1 / f^{\beta}$, then the ratio of $P$ measured at two different frequencies, $f_{1}$ and $f_{2}$, depends only on the ratio $f_{1} / f_{2}$ and not on the absolute value of $f_{1}$ or $f_{2}$ :

$$
P\left(f_{1}\right) / P\left(f_{2}\right)=\left(f_{2} / f_{1}\right)^{\beta} .
$$

This means that a dynamic that follows power-law scaling in its power spectrum contains no characteristic scale and thus is "scale free" (Mandelbrot, 1999; Schroeder, 1991).

Detrended fluctuation analysis. To more robustly investigate the scalefree behavior of the fMRI signal, we used the well-established detrended fluctuation analysis (DFA) method (Linkenkaer-Hansen et al., 2001; Eke et al., 2006), which was initially introduced to study correlations in DNA sequences (Peng et al., 1994). The DFA method has the particular advantage of being applicable to both stationary and nonstationary data. First, the signal is summed and the mean is subtracted:

$$
y_{j}=\sum_{i=1}^{j} x_{i}-\hat{\mu} .
$$

Then the local trend $y_{j, l}$ is estimated in non-overlapping windows of equal length $l$, using a least-squares fit to the data. For a given window size $l$, the fluctuation $F_{l}$ is determined as the root mean square variance on the local trend: 


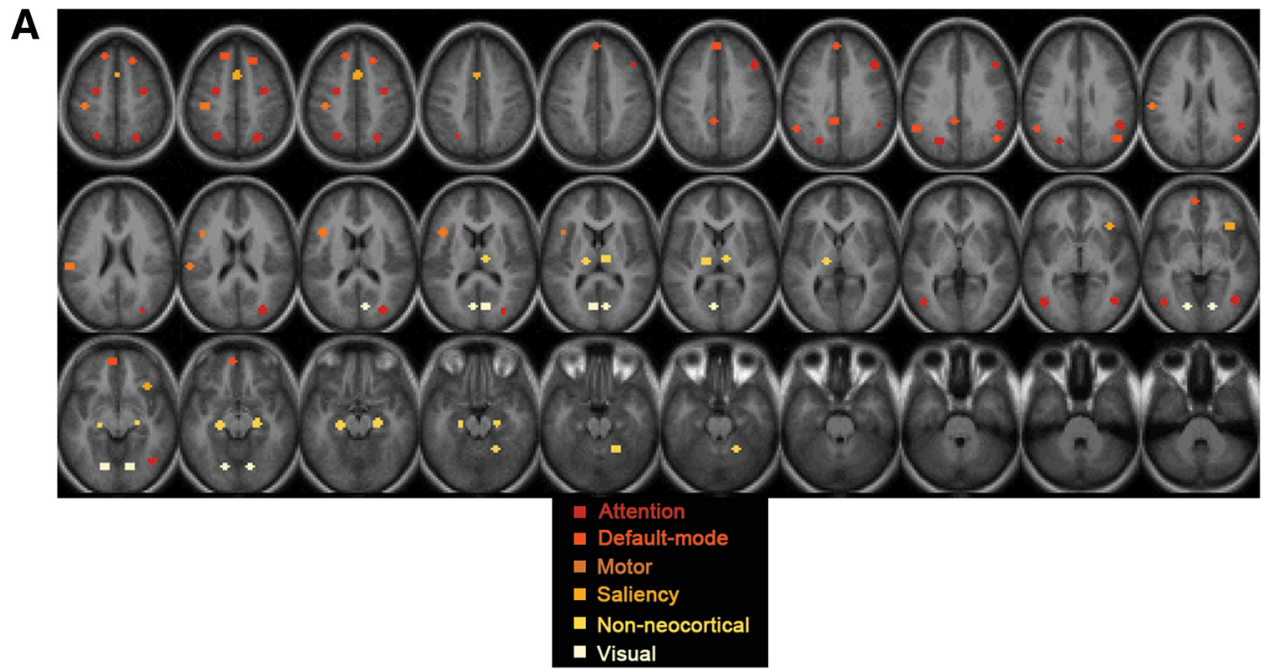

B

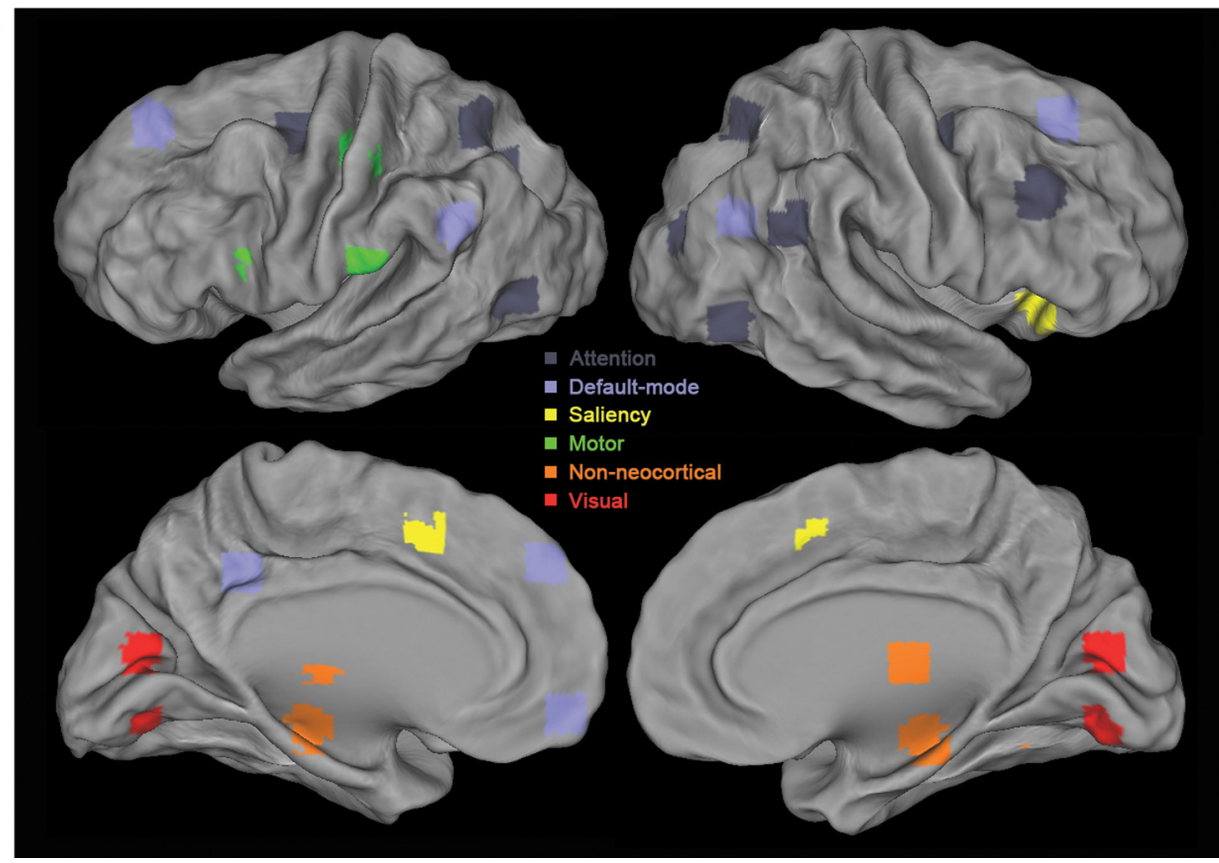

Figure 1. Locations of the ROls. The 31 ROIs, including 10 pairs of homologous brain regions, are color coded by their affiliated brain networks. A, Volume view. $B$, Surface view.

$$
F_{l}=\sqrt{\frac{1}{l} \sum_{j=1}^{l}\left(y_{j}-y_{j, l}\right)^{2} .}
$$

and then averaged across windows. For a scale-free time series, the fluctuation $F_{l}$ is related to window size $l$ by

$$
F_{l}=p l^{\alpha}
$$

or

$$
\log \left(F_{l}\right)=\log (p)+\alpha \log (l) .
$$

If $0<\alpha<1$, then $x$ is a stationary time series, and its Hurst exponent $H=$ $\alpha$. If $\alpha>1$, then $x$ is nonstationary, and its Hurst exponent $H=\alpha-1$ (Mandelbrot and Van Ness, 1968; Eke et al., 2002). The Hurst exponent $H$ characterizes the self-similarity of the integral of the original data:

$$
y_{i, n}={ }_{d} s^{-H} y_{i, s n}
$$

where $y_{i, n}$ is a sample time series of length $n, y_{i, s n}$ is a longer sample of the same process of length $s n$, and $={ }_{\mathrm{d}}$ means equal in distribution (Hurst, 1951).
To analyze our fMRI data, window lengths of 5, 10, 19, 38, and $95 \mathrm{fMRI}$ frames were chosen so that the number of frames in each run (190 after discarding the first four frames) is an integer multiple of the window length. The $\alpha$ of the fMRI signal in our data always resided within the region of $(0,1)$, indicating that the fMRI signal is stationary in first- and second-order statistics. Hence, the Hurst exponent was conveniently estimated by $\alpha$.

Goodness-of-fit test for scale invariance. To test how well scale invariance describes the fMRI signal from a particular brain region, we adapted a goodness-of-fit test developed for testing power-law distributions (Clauset et al., 2009). For each brain region, its time series (4 fMRI runs $\times 190$ frames for each behavioral condition) were extracted from each subject and subjected to DFA. The variance and Hurst exponent were computed and averaged across subjects. The Kolmogorov-Smirnov statistic was used to measure the distance $D$ between its $\log (F)-\log (l)$ plot and the best-fit linear-regression line in the log-log plot. Then, 1000 time series of fractional Gaussian noise (fGn) with the same length $(n=$ 760), variance, and Hurst exponent were generated using a circulant embedding algorithm (Helgason et al., 2011). Fractional Gaussian noise is a parsimonious model of stationary scale-free dynamics (Mandelbrot and Van Ness, 1968; Beran, 1994). Each synthetic fGn time series was 
Table 1. References, anatomical information, and scale-free goodness-of-fit tests for each region of interest (ROI), as well as the effect of behavioral condition (Rest versus Task) on fMRI signal variance, power-law exponent, and Hurst exponent (paired $t$ test; measurements from individual subjects were used as repeated measures, $n=17$ )

\begin{tabular}{|c|c|c|c|c|c|c|c|c|c|c|c|}
\hline \multirow[b]{2}{*}{ Network } & \multirow[b]{2}{*}{ ROI } & \multirow[b]{2}{*}{ Anatomical location } & \multirow[b]{2}{*}{ Talairach coordinates } & \multicolumn{2}{|c|}{$\begin{array}{l}\text { Goodness- } \\
\text { of-fit } p \\
\text { value } \\
\end{array}$} & \multicolumn{2}{|c|}{$\begin{array}{l}\text { Variance } \\
\text { rest versus task }\end{array}$} & \multicolumn{2}{|c|}{$\begin{array}{l}\text { Power-law exponent } \\
\text { rest versus task } \\
\end{array}$} & \multicolumn{2}{|c|}{$\begin{array}{l}\text { Hurst exponent } \\
\text { rest versus task }\end{array}$} \\
\hline & & & & Rest & Task & $t$ value & $p$ value & $t$ value & $p$ value & $t$ value & $p$ value \\
\hline \multirow[t]{6}{*}{ Attention } & vIPS $^{a}$ & $\begin{array}{l}\text { (L and R) ventral intra- } \\
\text { parietal sulcus }\end{array}$ & $-24,-69,30$ and $30,-80,16$ & 0.42 & 0.27 & 2.143 & $0.048^{*}$ & 3.194 & $0.005^{* * *}$ & 4.107 & $0.0008^{* * *}$ \\
\hline & $\mathrm{RTPJ}^{a, b}$ & $\begin{array}{l}\text { R temporoparietal } \\
\text { junction }\end{array}$ & $49,-50,28$ & 0.37 & 0.95 & 1.663 & 0.116 & 1.718 & 0.105 & 2.63 & $0.018^{*}$ \\
\hline & $\operatorname{RDLPFC}^{b, c, d}$ & $\begin{array}{l}\text { R dorsolateral prefron- } \\
\text { tal cortex }\end{array}$ & $43,22,34$ & 0.45 & 0.53 & 1.319 & 0.206 & 2.494 & $0.024^{*}$ & 2.014 & 0.06 \\
\hline & $\mathrm{pIPS}^{a}$ & $\begin{array}{l}\text { (L and R) posterior } \\
\text { intraparietal sulcus }\end{array}$ & $-25,-63,47$ and $23-6548$ & 0.17 & 0.55 & 1.268 & 0.223 & 2.733 & $0.015^{*}$ & 3.588 & $0.002^{* * *}$ \\
\hline & $\mathrm{MT}^{a}$ & $\begin{array}{l}\text { (L and } \mathrm{R}) \text { middle tem- } \\
\text { poral region }\end{array}$ & $-43,-70-3$ and $42-68-6$ & 0.56 & 0.48 & 0.947 & 0.358 & 3.592 & $0.002^{* * *}$ & 3.649 & $0.002^{* * *}$ \\
\hline & $\mathrm{FEF}^{a}$ & $\begin{array}{l}\text { ( } L \text { and } R \text { ) frontal eye } \\
\text { field }\end{array}$ & $-26,-9,48$ and $32,-9,48$ & 0.43 & 0.87 & 2.445 & $0.026^{*}$ & 2.816 & $0.012^{*}$ & 3.11 & $0.007^{* *}$ \\
\hline \multirow[t]{5}{*}{ Default } & $A G^{e}$ & ( $L$ and $R$ ) angular gyrus & $-51,-54,30$ and $45,-66,27$ & 0.21 & 0.51 & 3.22 & $0.005^{* * *}$ & 2.947 & $0.01^{* *}$ & 3.363 & $0.004^{* * *}$ \\
\hline & $\mathrm{SFG}^{e}$ & $\begin{array}{l}\text { (L and R) superior } \\
\text { frontal gyrus }\end{array}$ & $-15,33,48$ and $18,27,48$ & 0.80 & 0.98 & 2.334 & $0.033^{*}$ & 1.012 & 0.327 & 4.007 & $0.001^{* * *}$ \\
\hline & $\mathrm{PCC}$ & $\begin{array}{l}\text { Posterior cingulate } \\
\text { cortex }\end{array}$ & $-6,-45,33$ & 0.14 & 0.15 & 4.545 & $0.0003^{* * *}$ & 2.033 & 0.059 & 2.531 & $0.02^{*}$ \\
\hline & MPF $^{e}$ & $\begin{array}{l}\text { Medial prefrontal } \\
\text { cortex }\end{array}$ & $-6,51,-9$ & 0.65 & 0.54 & 1.423 & 0.174 & 2.296 & $0.035^{*}$ & 3.047 & $0.008^{* *}$ \\
\hline & $\mathrm{FP}^{e}$ & Frontopolar cortex & $-3,45,36$ & 0.72 & 0.09 & 2.679 & $0.016^{*}$ & 1.953 & 0.069 & 4.418 & $0.0004^{* * *}$ \\
\hline \multirow[t]{3}{*}{ Motor } & $\mathrm{LSII}^{a}$ & $\begin{array}{l}\text { L second somatosen- } \\
\text { sory area }\end{array}$ & $-57,-27,21$ & 0.98 & 0.28 & 1.063 & 0.303 & 1.902 & 0.075 & 2.6 & 0.019 \\
\hline & $\operatorname{Lmotor}^{a}$ & L primary motor cortex & $-39,-27,48$ & 0.19 & 0.79 & 3.377 & $0.004^{* * *}$ & 2.46 & $0.026^{*}$ & 4.487 & $0.0004^{* * *}$ \\
\hline & Broca $^{f}$ & Broca's area & $-42,13,14$ & 0.98 & 0.16 & 0.662 & 0.517 & 1.967 & 0.067 & 2.62 & $0.019^{*}$ \\
\hline \multirow[t]{3}{*}{ Non-neocortical } & Thalamus (Tha) ${ }^{a}$ & ( $\mathrm{L}$ and $\mathrm{R}$ ) thalamus & $-15,-21,6$ and $9,-18,9$ & 0.84 & 0.51 & 1.358 & 0.193 & 3.221 & $0.005^{* * *}$ & 1.327 & 0.2 \\
\hline & R Cerebellum $^{a}$ & R Cerebellum & $21,-54,-21$ & 0.96 & 0.32 & 0.927 & 0.368 & 1.938 & 0.07 & 2.353 & $0.03^{*}$ \\
\hline & $\mathrm{HF}^{g}$ & $\begin{array}{l}\text { (L and R) hippocampal } \\
\text { formation }\end{array}$ & $-21,-25,-14$ and $23,-23,-14$ & 0.90 & 0.44 & 2.159 & $0.046^{*}$ & 2.075 & $0.05^{*}$ & 2.731 & $0.015^{*}$ \\
\hline \multirow[t]{2}{*}{ Saliency } & $\mathrm{RFI}^{\mathrm{c}}$ & R frontoinsular cortex & $36,21,-6$ & 0.40 & 0.84 & 3.349 & $0.004^{* * *}$ & 2.596 & $0.02^{*}$ & 3.179 & $0.006^{* *}$ \\
\hline & $\mathrm{dACC}{ }^{b}$ & $\begin{array}{l}\text { Dorsal anterior cingu- } \\
\text { late cortex }\end{array}$ & $-1,10,46$ & 0.40 & 0.44 & 1.866 & 0.08 & 1.048 & 0.31 & 2.661 & $0.017^{*}$ \\
\hline \multirow[t]{2}{*}{ Visual } & vRetino $^{a}$ & $\begin{array}{l}\text { ( } L \text { and } R \text { ) ventral pri- } \\
\text { mary visual cortex }\end{array}$ & $-15,-75,-9$ and $15,-75,-9$ & 0.09 & 0.34 & 2.902 & $0.01^{* *}$ & 2.814 & $0.01^{* *}$ & 2.599 & $0.019^{*}$ \\
\hline & $\mathrm{dRetino}^{a}$ & $\begin{array}{l}\text { (L and R) dorsal pri- } \\
\text { mary visual cortex }\end{array}$ & $-6,-75,9$ and $9,-75,12$ & 0.33 & 0.19 & 2.414 & $0.028^{*}$ & 3.243 & $0.005^{* * *}$ & 2.694 & $0.016^{*}$ \\
\hline \multirow[t]{2}{*}{ Individual-defined } & $\mathrm{LMC}^{h}$ & L primary motor cortex & & & & 4.714 & $0.0002^{* * *}$ & 2.166 & $0.046^{*}$ & 4.053 & $0.0009^{* * *}$ \\
\hline & $\mathrm{RMC}^{h}$ & R primary motor cortex & & & & 3.303 & $0.004^{* * *}$ & 3.241 & $0.005^{* * *}$ & 2.849 & $0.012^{*}$ \\
\hline
\end{tabular}

${ }^{*} p<0.05 ;{ }^{* *} p<0.01 ;{ }^{* * *} p<0.005$

${ }^{a}$ He et al., 2007.

${ }^{b}$ Dosenbach et al., 2006.

'Seeley et al., 2007.

dVincent et al., 2008.

eShulman et al., 1997.

${ }^{f}$ Embick and Poeppel, 2006.

gVincent et al., 2006.

${ }^{h}$ Fox et al., 2007.

subjected to the same DFA, and the Kolmogorov-Smirnov statistic was used to measure the distance $D_{s}$ between its $\log (F)-\log (l)$ plot and its own best-fit linear-regression line. The $p$ value is defined as the fraction of synthetic time series with $D_{s}$ that is larger than the original $D$ of the fMRI time series. The larger the $p$ value, the more plausible the fGn model is for representing the original fMRI time series, and the better the fit of the original data to a scale-free distribution. The hypothesis that the fMRI signal is scale free is ruled out if $p<0.05$.

\section{Results}

fMRI power-law exponent varies across brain networks, correlates with variance and glucose metabolism

Thirty-one ROIs were defined based on our previous functional studies or published articles, covering key nodes of five known brain networks (attention, default-mode, motor, saliency, and visual) and three additional regions outside the neocortex (hippocampus, thalamus, and cerebellum) (Fig. 1, Table 1). The fMRI power-law exponent, variance, and metabolic values were extracted from each ROI and then averaged across 10 pairs of homologous ROIs, resulting in 21 brain regions in total.

In our previous study (He et al., 2010), fMRI signals were passed through conventional functional connectivity preprocessing (Fox and Raichle, 2007) (spatial smoothing, mean subtraction, regression of head motion, ventricular, white matter, and whole-brain signals) before power spectral calculation. Here, to stay closer to the raw fMRI signal, we subjected it only to mean subtraction and regression of head motion (see Materials and 
Methods, fMRI data preprocessing) and reproduced previous results (Fig. 2A). The spontaneous fMRI signal follows a power-law distribution in its temporal power spectrum: the power-law fit $P(f)=$ $a / f^{\beta}$ is highly significant $(p<0.0001$, power-law regression) for all 21 regions (Fig. 2A).

Next, we compared the fit of several alternative models to the fMRI power spectrum. The power spectrum from each brain region was fit (using least-squares fitting) with (1) an exponential function, (2) a log-normal function, (3) a powerlaw function, and (4) a power-law function using only the $<0.1 \mathrm{~Hz}$ frequency region to avoid aliasing artifact in higher frequencies (see Materials and Methods, Power spectrum calculation). The Kolmogorov-Smirnov distance $D$ between the original power spectrum and the fitted function was computed and subjected to paired $t$ tests between different models (Fig. $2 B$, with brain regions as repeated measures). The power-law functions provided better fit to the data than exponential or log-normal function. Power-law function using only the $<0.1 \mathrm{~Hz}$ frequency region provided significantly better fit than exponential $(p<0.05)$, $\log$-normal $(p=0.0005)$, or power-law function using the entire frequency region $(p=0.02)$.

We thus obtained power-law exponent $\beta$ by fitting a powerlaw function to the $<0.1 \mathrm{~Hz}$ frequency region of the power spectrum. The power-law exponent $\beta$ ranged from 0.62 (left second somatosensory area) to 1.14 (ventral primary visual cortex). The mean of $\beta$ across 21 brain regions was 0.84 . Power-law exponent $\beta$ was significantly different across brain networks (Fig. $2 C, p=$ 0.0076, assessed by an ANOVA), being largest in visual, saliency, and default-mode networks and smallest in motor network and non-neocortical regions. In contrast, the variance of the fMRI signal did not differentiate between brain networks ( $p>0.5$, assessed by an ANOVA). Nevertheless, there was a significant correlation between $\mathrm{fMRI}$ signal variance and power-law exponent (Fig. $3 A, r=0.517$, $p=0.015)$.

Interestingly, among brain metabolic values, CMRGlu ( $p=$ $0.007)$, GI $(p<0.0001)$, and OEF $(p=0.006)$ also differentiated between brain networks (Fig. $2 D$, assessed by ANOVA). Hence, OEF is not as uniform across the brain as suggested previously (Raichle et al., 2001). Nonetheless, OEF in the default-mode network was close to the whole-brain mean ( $1.4 \%$ lower, $p>0.4)$, confirming a previous conclusion that it is not spontaneously active at rest (Raichle et al., 2001). The networks with largest deviation of OEF from the whole-brain mean are the saliency network comprising the dorsal anterior cingulate cortex and frontoinsular cortex $(5.5 \%$ lower than the whole-brain mean, implying spontaneous activation, $p<0.05$ ) and the visual cortex (10.4\% higher than the whole-brain mean, implying deactivation likely attributable to eye closure during these scans, $p<0.0001)$.

Correlating the fMRI power-law exponent and variance with brain metabolic values, we found a significant correlation between power-law exponent and CMRGlu $(r=0.514$, $p=0.016)($ Fig. $3 B)$. No significant correlation between fMRI
B
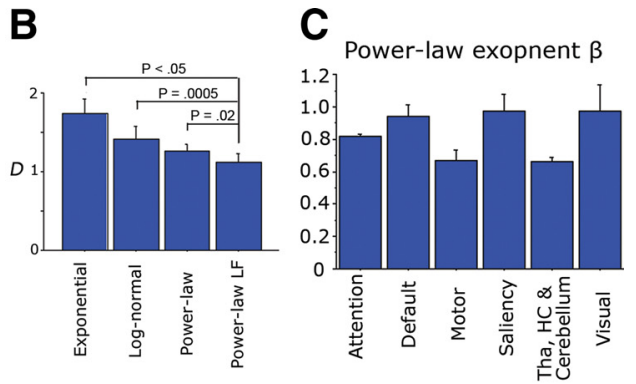

OEF
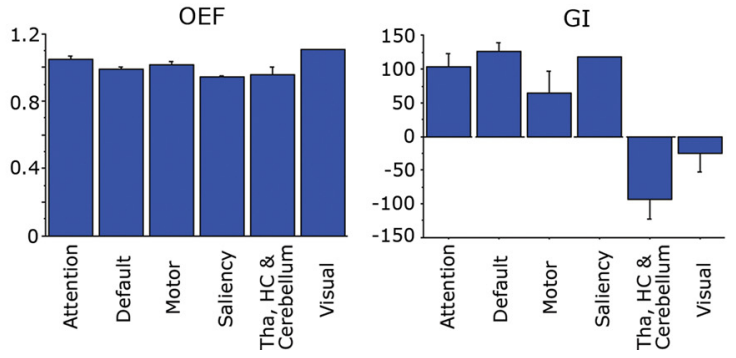

Figure 2. Power spectra of spontaneous fMRI signals and the variations of power-law exponent and metabolic values across log-normal, power-law, and power-law using only the low-frequency $(<0.1 \mathrm{~Hz})$ (Power-law LF) region. The Kolmogorov-Smirtests were used to compare different models. $C$ Variation of power-law exponent across brain networks ( $p=0.0076$, assessed by an ANOVA). $\boldsymbol{D}$, Variations of CMRGlu $(p=0.007), 0 \mathrm{EF}(p=0.006)$, and $\mathrm{GI}(p<0.0001)$ across brain networks, assessed by ANOVA. HC, Hippocampus; Tha, thalamus. Error bars denote SEM. For other abbreviations, see Table 1.
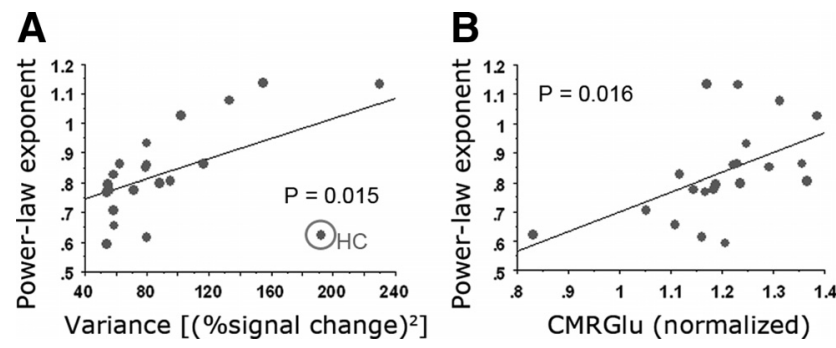

Figure 3. fMRI power-law exponent correlates with variance and CMRGlu. $A$, A scatter plot showing the values of power-law exponent and variance of the $\mathrm{FMRI}$ signal for each of $21 \mathrm{brain}$ regions (correlation, $r=0.517, p=0.015$ ). The best-fit linear-regression line is shown. The outlier indicated by circle is the hippocampal formation $(\mathrm{HC})$, with a very high variance but small power-law exponent. The $r$ and $p$ values were computed including the hippocampal formation. $\boldsymbol{B}$, A scatter plot showing fMRI signal power-law exponent and regional CMRGlu value for each of 21 brain regions $(r=0.514, p=0.016)$.

signal variance and any of the metabolic values was found (all $p>0.2)$.

\section{The fMRI signal is scale free; its Hurst exponent correlates tightly with power-law exponent}

Although power-law scaling provides a good fit to the fMRI power spectrum (Fig. 2A,B), to rigorously test the hypothesis that fMRI signals are scale free, we applied a more robust time-domain method, DFA, which complements the above frequency-domain approach, and then used goodness-of-fit tests to examine how well scale invariance describes fMRI signals.

The DFA procedure measures the amount of fluctuation $F$ of detrended integrated signal at different length scales, thereby revealing the scaling properties of the signal. If the signal is scale 


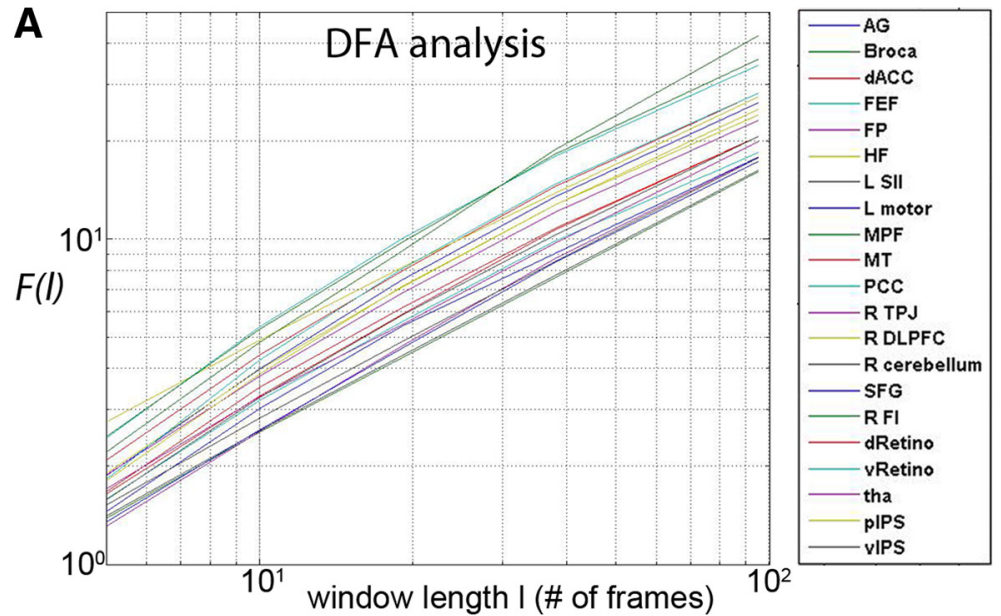

C

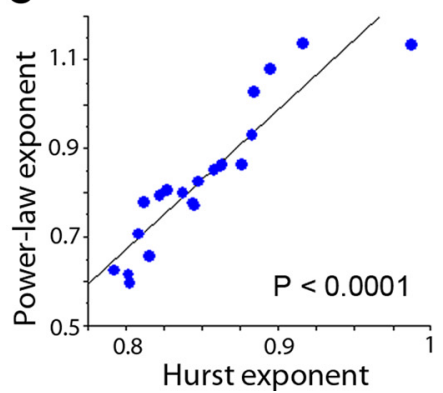

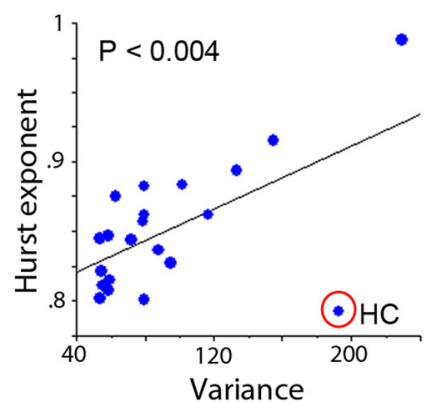

Hurst exponent

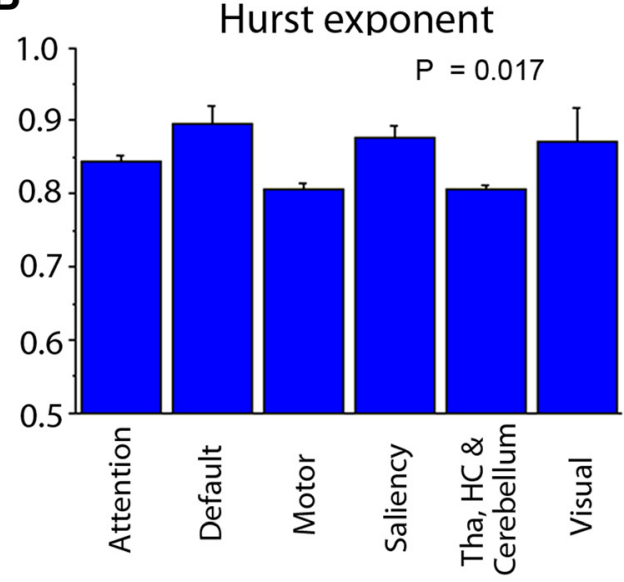

D

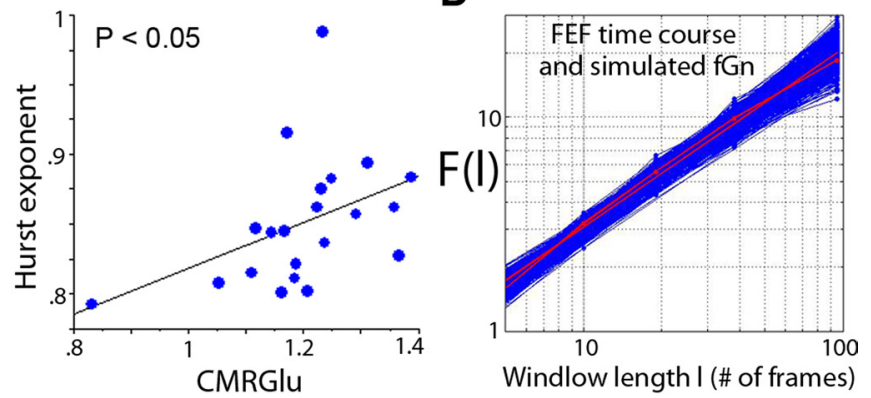

Figure 4. DFA and Hurst exponent of spontaneous fMRI signals. $A$, DFA plots for 21 brain regions, showing fluctuation $(F)$ measured at different window lengths $(I)$ plotted in double-logarithmic scales. $\boldsymbol{B}$, Variation of Hurst exponent across brain networks, assessed by an ANOVA $(p=0.017)$. C, Hurst exponent correlates with power-law exponent $\left(r=0.92, p<10^{-8}\right)$, variance $(r=0.59$, $p<0.004)$, and CMRGlu $(r=0.44, p<0.05)$. D, Goodness-of-fit test for one example region—frontal eye field (FEF) — on its fit to a scale-free distribution $(p=0.43)$. The red line with dots is the raw $\mathrm{F}-/ \mathrm{plot}$ for the $\mathrm{FEF}$ time series, and the red line without marker is its least-squares power-law fit. The blue lines are the $\mathrm{F}-\mathrm{I}$ plot for 1000 synthetic fGn time series with the same variance and Hurst exponent as the FEF time series and their respective least-squares power-law fit. HC, Hippocampus. For other abbreviations, see Table 1.

invariant, then $F$ scales with length scale $l$ in a power-law manner: $F_{l}=p l^{\alpha}$, and $\log (F)$ is linearly related to $\log (l)$. The slope $\alpha$ of the $\log (F)-\log (l)$ plot estimates the Hurst exponent $H$, the single parameter that describes the scale-invariant properties of a selfsimilar signal (see Materials and Methods, Detrended fluctuation analysis).

The $\log (F)-\log (l)$ plots obtained by DFA follow an approximately straight line for all 21 brain regions (Fig. $4 A$ ), indicating scale invariance. Goodness-of-fit tests suggested that scale invariance is a good description for all 21 brain regions (Fig. $4 D$, Table 1 , all $p>0.05$; with the exception of one region, all $p>0.1$ ).

From the DFA plots, Hurst exponent $H$ was estimated for each of 21 brain regions. $H$ was tightly correlated with the power-law exponent estimated from the power spectrum (Fig. 4C, left, $r=$ 0.92, $p<10^{-8}$ ). It also reproduced all of the results obtained using the power-law exponent $\beta$ (Figs. $2 C, 3$ ): $H$ differentiated between brain networks (Fig. $4 B, p=0.017$, ANOVA) and correlated with $\mathrm{fMRI}$ signal variance (Fig. $4 C$, middle, $r=0.59, p<$ 0.004 ) and brain glucose metabolism (Fig. $4 C$, right, $r=0.44, p<$ 0.05 ) but not with the other brain metabolic measurements.

\section{The effect of task performance on fMRI signal variance,} power-law exponent, and Hurst exponent

Next, we investigated the effect of task performance on fMRI signal variance and scale-free properties. Goodness-of-fit tests on the DFA measurements suggested that the fMRI signals in all 21 brain regions remain scale free during task performance (all $p>$ 0.05 ; with the exception of one region, all $p>0.1$; see Table 1 ). Remarkably, across 21 brain regions covering all lobes and five major brain networks, fMRI signal power-law exponent, Hurst exponent and variance decreased in every brain region during task performance (Fig. 5A). The effect of task performance on variance $(p=0.0003)$, power-law exponent $\left(p<10^{-8}\right)$, and Hurst exponent $(p<0.0005)$ were all highly significant (paired $t$ tests across all brain regions). To test which brain regions had a significant decrease in these measurements by themselves, we performed a paired $t$ test for each brain region using individual subjects' data as repeated measures $(n=17)$ and found a significant task effect on variance in 11 of 21 regions, on power-law exponent in 13 of 21 regions, and on Hurst exponent in 19 of 21 regions at $p<0.05$ level (uncorrected; Table 1 ). The only two brain regions whose Hurst exponent did not change significantly under task performance were right DLPFC $(p=0.06)$ and thalamus $(p=0.2)$.

Notably, the power-law exponent, Hurst exponent, and variance of the fMRI signal decreased not only in task-activated brain areas, including the visual, motor, attention, and saliency networks, but also in task-deactivated default-mode network (for task activation/deactivation maps from two typical subjects, see Fig. 6). This finding parallels previous results showing that fMRI signal variance not only decreased in visual cortex during eyesopen condition compared with the eyes-closed condition (McAvoy et al., 2008; Bianciardi et al., 2009) but also decreased in default-mode network during deactivation under a working memory task (Fransson, 2006).

We further defined in each subject the LMC and its homologous RMC based on task activation and functional connectivity, respectively (see Materials and Methods, Definitions of regions of 


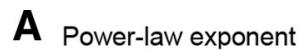

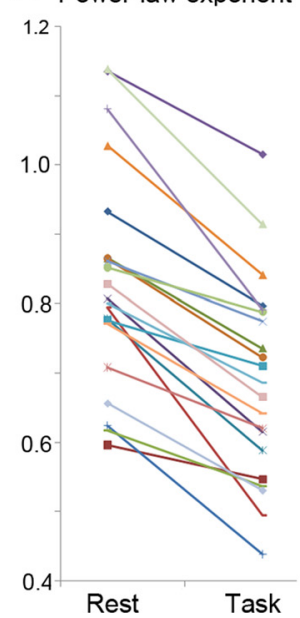

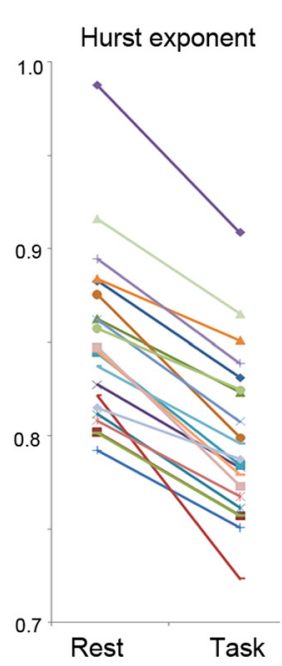

B

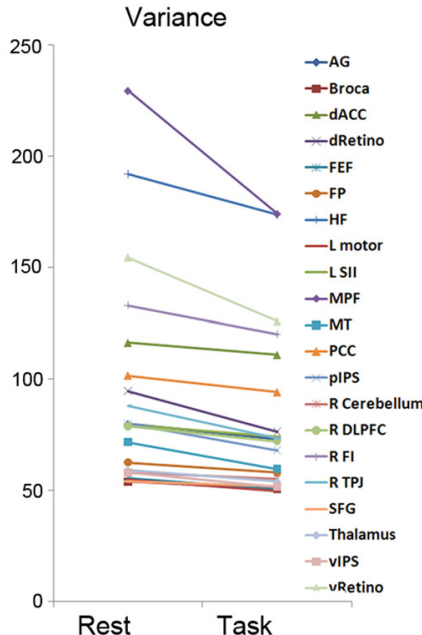

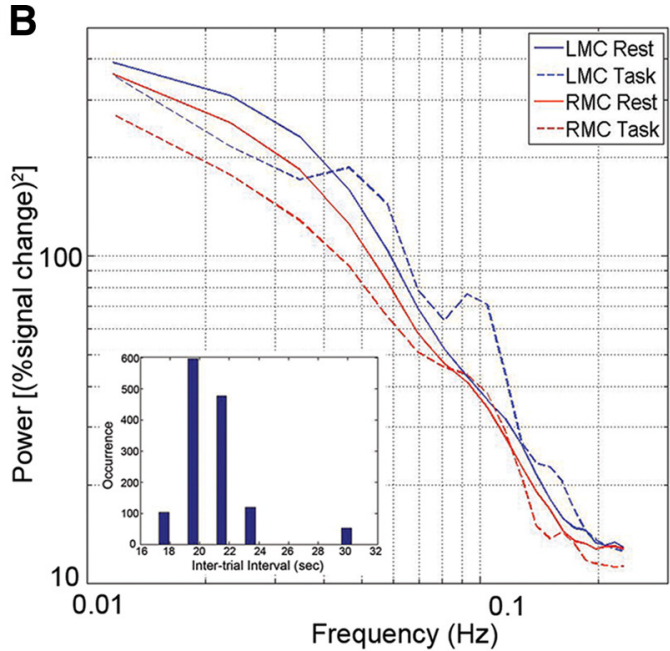

Figure 5. The effect of task performance on $\mathrm{fMRI}$ signal variance and scale-free properties. $A$, fMRI signal power-law exponent (left), Hurst exponent (middle), and variance (right) for each of 21 brain regions during rest and task. For statistical results on the effect of task, see Results and Table 1. B, fMRI signal power spectra during rest and task (without normalization by total power) were computed for the LMC and RMC regions individually defined for each subject and then averaged across 17 subjects. Significant decrease of variance, power-law exponent, and Hurst exponent were found in both regions (for values, see Results and Table 1). Inset, The distribution of intertrial intervals of the button-press task. For abbreviations, see Table 1.

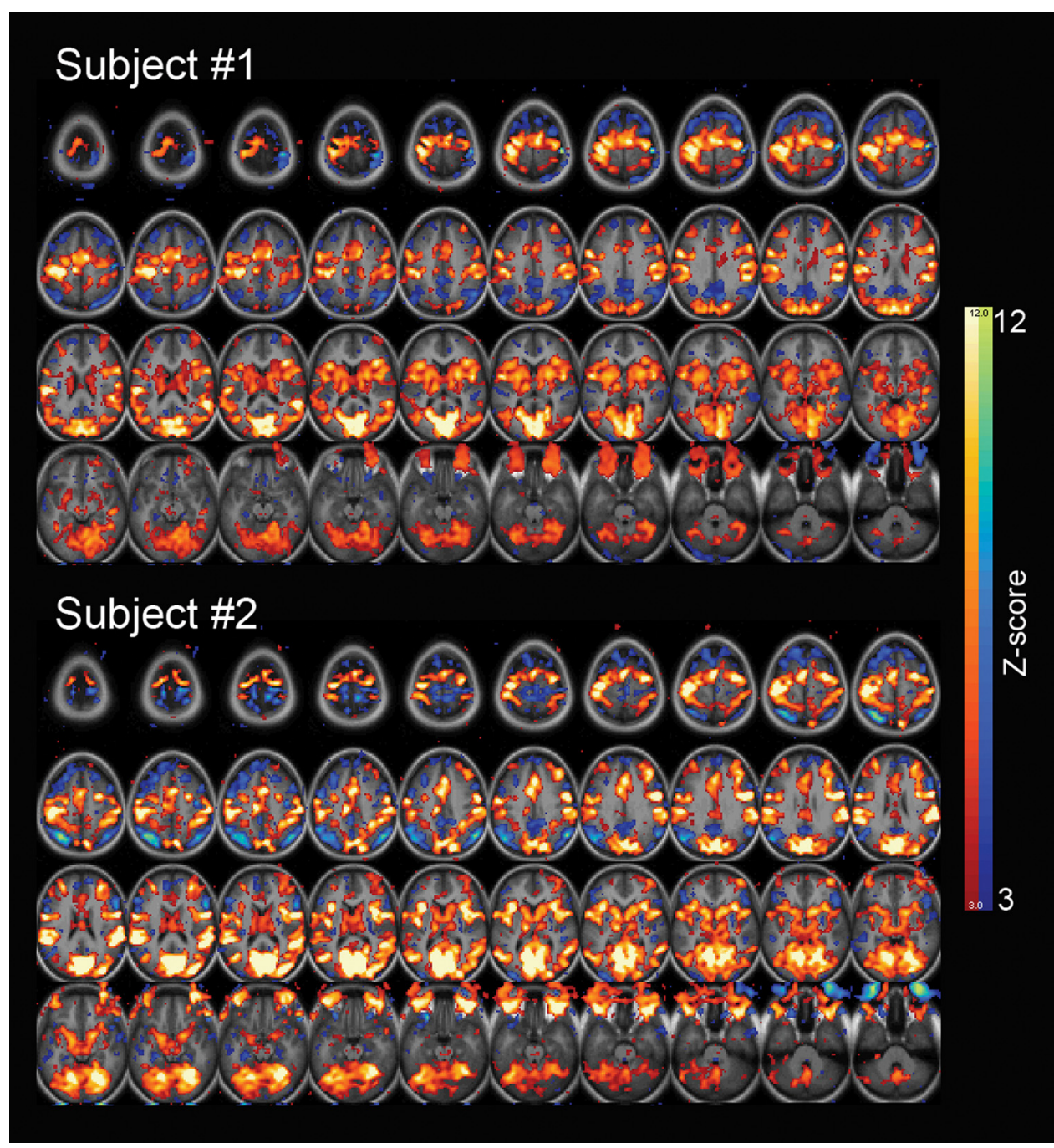

Figure 6. Task-induced activation and deactivation patterns from two example subjects. A general-linear model with an event-related design was used to generate $t$-score maps, which were converted to equally probable $Z$-score maps, and thresholded at $Z=3$ for both activations (shown in red-yellow) and deactivations (shown in blue-green). 
interest). Across all subjects, all three measurements-fMRI signal variance, power-law exponent, and Hurst exponent-decreased in both regions during task (variance: LMC, $p=0.0002$; $\mathrm{RMC}, p=0.004$; power-law exponent: LMC, $p<0.05$; $\mathrm{RMC}, p=$ 0.005; Hurst exponent: LMC, $p=0.0009$; RMC, $p=0.01$, paired $t$ tests). The power spectra of these two regions during rest and task are shown in Figure 5B. During task, there are two discernable peaks at $\sim 0.05$ and $0.1 \mathrm{~Hz}$ in the power spectrum of LMC. Although the onset of the stimulus was unpredictable to the subjects, the distribution of the intertrial intervals had a peak at 20-22 s (Fig. $5 B$, inset), corresponding to $0.05 \mathrm{~Hz}$ (the $0.1 \mathrm{~Hz}$ peak is its harmonic). This suggests that implicit structure in task design, unbeknownst to the subject, can nevertheless entrain the fMRI signal into regular oscillations.

\section{Discussion}

Scale-free dynamics are present across many different spatiotemporal scales in the brain: from neurotransmitter release (Lowen et al., 1997), neuronal spike trains (Grüneis et al., 1989; Lowen et al., 2001), network firing rates (Garcia-Perez et al., 2007; Mazzoni et al., 2007), field potentials (Freeman and Zhai, 2009; Manning et al., 2009; Miller et al., 2009; Milstein et al., 2009; He et al., 2010), to fMRI signals. They are also prevalent in the cognition and behaviors produced by the brain, such as human performance fluctuations (Gilden, 2001), music and speech (Voss and Clarke, 1975), and fruit fly flight behaviors (Maye et al., 2007). However, their spatiotemporal structures, functional properties, and underlying mechanisms have so far remained elusive.

Here we have shown that fMRI signals belong to the class of scale-free brain activity. This is in line with its recently discovered correlation with the slow cortical potential (SCP) (He et al., 2008; He and Raichle, 2009), the low-frequency end of scale-free brain field potentials (He et al., 2010). The current results, showing that scale-free properties of the fMRI signal differentiate between brain networks, correlate with brain glucose metabolism and alter during task performance strongly suggest the functional significance and neuronal origins of scale-free fMRI signals. Furthermore, whereas electrical field potentials are a combination of scale-free brain activity and brain oscillations (He et al., 2010), the spontaneous fMRI signal does not seem to contain any oscillation but rather is composed mainly of scale-free activity, like its electrical counterpart SCP.

Importantly, the current findings mirror previous electrophysiological observation that the power-law exponent and variance of the SCP decrease in task-activated brain regions (He et al., 2010), reinforcing the correlation between the fMRI signal and the SCP (He and Raichle, 2009). Larger power-law exponent suggests higher time-lagged autocorrelation, indicating that the past dynamics of the system has stronger influence on its future dynamics, i.e., the system has more long-range memory (Mandelbrot and Van Ness, 1968; Eke et al., 2002). In contrast, smaller power-law exponent suggests that the system has less temporal redundancy and is more efficient in online information processing. Hence, these findings fit well with the need for the system to process incoming information in an activated state and the need to maintain memory and plan for future during the baseline state (Berkes et al., 2011). In remarkable parallel to these physiological findings, fluctuations in human behavioral performance during psychological tasks also exhibit a scale-free power spectrum (Gilden, 2001), and the power-law exponent decreases with increasing task difficulty (Clayton and Frey, 1997). Thus, previous findings showing that patients with more anxiety had smaller fMRI signal power-law exponent (Tolkunov et al., 2010) can be
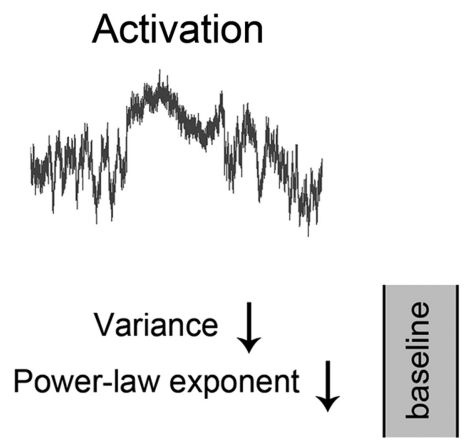

\section{Deactivation}
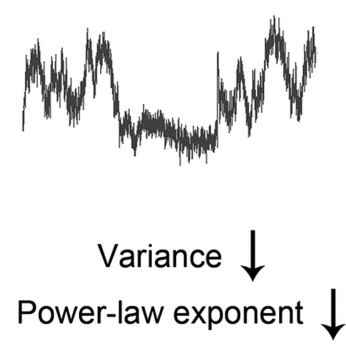

Figure 7. Schematic showing the effect of task on fMRI signals. The present results reveal that the fMRI signal variance, power-law exponent, and Hurst exponent decrease in both taskactivated and -deactivated brain regions, suggesting that the dynamic range and long-range memory of the $\mathrm{fMRI}$ signal are largest during the baseline resting state. The curves in the top were modeled using Gaussian-distributed white noise filtered in the frequency domain by $P \propto$ $1 / f^{\beta}$, with $\beta$ equal to the power-law exponent averaged across the 21 brain regions (rest, 0.83 ; task, 0.69). The variance during activation/deactivation was modeled as $97.8 \%$ of that during rest (average value across 21 brain regions).

interpreted as these patients' brains being constantly activated in light of the present results (note that this study published power spectrum of differentiated fMRI signal, which decreases $\beta$ by 2). Similarly, previous results showing that fMRI signal Hurst exponent is larger in patients with Alzheimer's disease (AD) than in age-matched controls (Maxim et al., 2005) implicates that these patients' brains are less efficient at processing information (note that "long-range memory" discussed herein refers to time-lagged autocorrelation at the range of seconds to minutes and do not correspond to episodic memory encoded in the structures of neuronal networks that is disrupted in $\mathrm{AD}$ ).

We found that fluctuations and long-range memory of the fMRI signal are largest during baseline condition and, moving away from this baseline-either activation or deactivation-attenuates the fluctuation and reduces long-range memory, indicating that the system has an optimal dynamic range (Fig. 7). This is consistent with the observations that fMRI signal variance not only decreases in the default-mode network during deactivation under a working memory task (Fransson, 2006) but also decreases in visual cortex during activation (McAvoy et al., 2008; Bianciardi et al., 2009). Our results extend these previous findings by showing that a reduction of the memory span in the fMRI signal accompanies the task-induced reduction of variance.

Such widespread changes in fMRI signal variance and powerlaw exponent contrast with the more localized change in the power-law exponent of brain electrical field potentials under a similar task, which occurred mainly in visual and motor areas (He et al., 2010). The exact reason for this difference is yet unclear, but it resonates with the impression in the literature that measurements from electrophysiological signals are often more localized than fMRI signals under similar types of tasks (Sirotin and Das, 2009; Ojemann, 2010). An intriguing possibility is that the fMRI signal, more than electrical field potentials, reflects a behavioral state change. Presently, it is unclear what happens to the variance and power-law exponent of the SCP in default-mode network under task-induced deactivation. Filling in this gap would help reveal the extent to which the fMRI signal follows brain field potentials.

The power-law exponent of the fMRI signal being $<1$ is an indication of stationarity in the first- and second-order statistics (Mandelbrot and Van Ness, 1968; Eke et al., 2002). This is corroborated by our DFA, which revealed $\alpha$ to be $<1$ for all brain 
regions, again suggesting that the fMRI signal is stationary (Eke et al., 2002). In contrast, brain electrical field potentials have a power-law exponent that is in the range of 2-4 (Freeman and Zhai, 2009; Miller et al., 2009; Milstein et al., 2009; He et al., 2010), signifying nonstationarity, which is a common impression of brain electrophysiological signals (Niedermeyer and Lopes da Silva, 2005). This notable difference between fMRI signals and brain electrical field potentials argues that future models of neurovascular coupling should take into account the transformation from nonstationarity to stationarity. Crucially, this cannot be achieved by conventional convolution with a hemodynamic response function, which is equivalent to a simple low-pass filter in the frequency domain.

In summary, we have shown that the fMRI signal recorded from the human brain belongs to the class of scale-free dynamics, and its scale-free properties alter with the functional state of the brain. During rest, the power-law exponent of the fMRI signal differentiates among brain networks and correlates with brain glucose metabolism. During task state, the variance and longrange memory of the fMRI signal decrease across widespread brain regions, suggesting that the dynamic range of the fMRI signal is largest and the temporal memory is longest during rest. The decrease of long-range memory during task is consistent with more efficient online information processing. Such changes in temporal memory contained in the fMRI signal and brain electrical field potentials between rest and task are suggestive of corresponding processes at the cellular/synaptic or neuronal network level, which strongly invite future investigation. We here propose that decorrelation or decoupling among neuronal groups during task state (e.g., Poulet and Petersen, 2008) may translate into decreased temporal memory recorded in field potentials or fMRI signals. We eagerly anticipate future empirical and modeling tests of this hypothesis. Last but not least, the stationarity of the fMRI signal on the one hand and the nonstationarity of brain field potentials on the other hand, revealed by their respective scale-free parameters, argue that future models of neurovascular coupling should take into account the transformation from a nonstationary signal to a stationary one.

\section{References}

Bak P (1996) How nature works. New York: Springer.

Beran J (1994) Statistics for long-memory processes. Boca Raton, FL: Chapman and Hall/CRC.

Berkes P, Orbán G, Lengyel M, Fiser J (2011) Spontaneous cortical activity reveals hallmarks of an optimal internal model of the environment. Science 331:83-87.

Bianciardi M, Fukunaga M, van Gelderen P, Horovitz SG, de Zwart JA, Duyn JH (2009) Modulation of spontaneous fMRI activity in human visual cortex by behavioral state. Neuroimage 45:160-168.

Biswal B, Yetkin FZ, Haughton VM, Hyde JS (1995) Functional connectivity in the motor cortex of resting human brain using echo-planar MRI. Magn Reson Med 34:537-541.

Bullmore E, Long C, Suckling J, Fadili J, Calvert G, Zelaya F, Carpenter TA, Brammer M (2001) Colored noise and computational inference in neurophysiological (fMRI) time series analysis: resampling methods in time and wavelet domains. Hum Brain Mapp 12:61-78.

Bullmore E, Fadili J, Maxim V, Sendur L, Whitcher B, Suckling J, Brammer M, Breakspear M (2004) Wavelets and functional magnetic resonance imaging of the human brain. Neuroimage 23 [Suppl 1]:S234-S249.

Ciuciu P, Varoquaux G, Abry P, Almog M (2011) Multifractal analysis of resting state networks in functional MRI. Presented at the Eighth Meeting of the IEEE International Symposium on Biomedical Imaging, Chicago, March to April.

Clauset A, Shalizi CR, Newman MEJ (2009) Power-law distributions in empirical data. SIAM Rev 51:661-703.

Clayton K, Frey B (1997) Studies of mental "noise." Nonlinear Dynamics Psychol Life Sci 1:173-180.
Damoiseaux JS, Rombouts SA, Barkhof F, Scheltens P, Stam CJ, Smith SM, Beckmann CF (2006) Consistent resting-state networks across healthy subjects. Proc Natl Acad Sci U S A 103:13848-13853.

Dosenbach NU, Visscher KM, Palmer ED, Miezin FM, Wenger KK, Kang HC, Burgund ED, Grimes AL, Schlaggar BL, Petersen SE (2006) A core system for the implementation of task sets. Neuron 50:799-812.

Dosenbach NU, Fair DA, Miezin FM, Cohen AL, Wenger KK, Dosenbach RA, Fox MD, Snyder AZ, Vincent JL, Raichle ME, Schlaggar BL, Petersen SE (2007) Distinct brain networks for adaptive and stable task control in humans. Proc Natl Acad Sci U S A 104:11073-11078.

Eke A, Herman P, Kocsis L, Kozak LR (2002) Fractal characterization of complexity in temporal physiological signals. Physiol Meas 23:R1-R38.

Eke A, Hermán P, Hajnal M (2006) Fractal and noisy CBV dynamics in humans: influence of age and gender. J Cereb Blood Flow Metab 26:891-898.

Embick D, Poeppel D (2006) Mapping syntax using imaging: prospects and problems for the study of neurolinguistic computation. In: Encyclopedia of language and linguistics (Brown K, ed), pp 484-486. Oxford: Elsevier.

Fox MD, Raichle ME (2007) Spontaneous fluctuations in brain activity observed with functional magnetic resonance imaging. Nat Rev Neurosci 8:700-711.

Fox MD, Snyder AZ, Vincent JL, Corbetta M, Van Essen DC, Raichle ME (2005) The human brain is intrinsically organized into dynamic, anticorrelated functional networks. Proc Natl Acad Sci U S A 102:9673-9678.

Fox MD, Corbetta M, Snyder AZ, Vincent JL, Raichle ME (2006) Spontaneous neuronal activity distinguishes human dorsal and ventral attention systems. Proc Natl Acad Sci U S A 103:10046-10051.

Fox MD, Snyder AZ, Vincent JL, Raichle ME (2007) Intrinsic fluctuations within cortical systems account for intertrial variability in human behavior. Neuron 56:171-184.

Fransson P (2006) How default is the default mode of brain function? Further evidence from intrinsic BOLD signal fluctuations. Neuropsychologia 44:2836-2845.

Freeman WJ, Zhai J (2009) Simulated power spectral density (PSD) of background electrocorticogram (ECoG). Cogn Neurodyn 3:97-103.

Friston KJ (2009) Modalities, modes, and models in functional neuroimaging. Science 326:399-403.

Garcia-Perez E, Mazzoni A, Torre V (2007) Spontaneous electrical activity and behavior in the leech hirudo medicinalis. Front Integr Neurosci 1:8.

Garrett DD, Kovacevic N, McIntosh AR, Grady CL (2010) Blood oxygen level-dependent signal variability is more than just noise. J Neurosci 30:4914-4921.

Garrett DD, Kovacevic N, McIntosh AR, Grady CL (2011) The importance of being variable. J Neurosci 31:4496-4503.

Gilden DL (2001) Cognitive emissions of 1/f noise. Psychol Rev 108:33-56.

Goldberger AL, Amaral LA, Hausdorff JM, Ivanov P, Peng CK, Stanley HE (2002) Fractal dynamics in physiology: alterations with disease and aging. Proc Natl Acad Sci U S A 99 [Suppl 1]:2466-2472.

Grüneis F, Nakao M, Yamamoto M, Musha T, Nakahama H (1989) An interpretation of $1 / \mathrm{f}$ fluctuations in neuronal spike trains during dream sleep. Biol Cybern 60:161-169.

Haxby JV, Gobbini MI, Furey ML, Ishai A, Schouten JL, Pietrini P (2001) Distributed and overlapping representations of faces and objects in ventral temporal cortex. Science 293:2425-2430.

Haynes JD, Rees G (2006) Decoding mental states from brain activity in humans. Nat Rev Neurosci 7:523-534.

He BJ, Raichle ME (2009) The fMRI signal, slow cortical potential and consciousness. Trends Cogn Sci 13:302-309.

He BJ, Snyder AZ, Vincent JL, Epstein A, Shulman GL, Corbetta M (2007) Breakdown of functional connectivity in frontoparietal networks underlies behavioral deficits in spatial neglect. Neuron 53:905-918.

He BJ, Snyder AZ, Zempel JM, Smyth MD, Raichle ME (2008) Electrophysiological correlates of the brain's intrinsic large-scale functional architecture. Proc Natl Acad Sci U S A 105:16039-16044.

He BJ, Zempel JM, Snyder AZ, Raichle ME (2010) The temporal structures and functional significance of scale-free brain activity. Neuron 66:353-369.

Helgason H, Pipiras V, Abry P (2011) Fast and exact synthesis of stationary multivariate Gaussian time series using circulant embedding. Signal Processing 91:1123-1133.

Hurst H (1951) Long-term storage capacity of reservoirs. Am Soc Civ Eng 116:770-808 
Jenkins GM, Watts DG (1998) Spectral analysis and its applications, Ed 5. Boca Raton, FL: Emerson-Adams.

Linkenkaer-Hansen K, Nikouline VV, Palva JM, Ilmoniemi RJ (2001) Long-range temporal correlations and scaling behavior in human brain oscillations. J Neurosci 21:1370-1377.

Lowen SB, Cash SS, Poo M, Teich MC (1997) Quantal neurotransmitter secretion rate exhibits fractal behavior. J Neurosci 17:5666-5677.

Lowen SB, Ozaki T, Kaplan E, Saleh BE, Teich MC (2001) Fractal features of dark, maintained, and driven neural discharges in the cat visual system. Methods 24:377-394.

Mandelbrot BB (1999) Multifractals and 1/f noise: wild self-affinity in physics. New York: Springer.

Mandelbrot BB, Van Ness JW (1968) Fractional Brownian motions, fractional noises and applications. SIAM Rev 10:422-437.

Manning JR, Jacobs J, Fried I, Kahana MJ (2009) Broadband shifts in local field potential power spectra are correlated with single-neuron spiking in humans. J Neurosci 29:13613-13620.

Maxim V, Sendur L, Fadili J, Suckling J, Gould R, Howard R, Bullmore E (2005) Fractional Gaussian noise, functional MRI and Alzheimer's disease. Neuroimage 25:141-158.

Maye A, Hsieh CH, Sugihara G, Brembs B (2007) Order in spontaneous behavior. PLoS One 2:e443.

Mazzoni A, Broccard FD, Garcia-Perez E, Bonifazi P, Ruaro ME, Torre V (2007) On the dynamics of the spontaneous activity in neuronal networks. PLoS One 2:e439.

McAvoy M, Larson-Prior L, Nolan TS, Vaishnavi SN, Raichle ME, d'Avossa G (2008) Resting states affect spontaneous BOLD oscillations in sensory and paralimbic cortex. J Neurophysiol 100:922-931.

Miller KJ, Sorensen LB, Ojemann JG, den Nijs M (2009) Power-law scaling in the brain surface electric potential. PLoS Comput Biol 5:e1000609.

Milstein J, Mormann F, Fried I, Koch C (2009) Neuronal shot noise and Brownian 1/f2 behavior in the local field potential. PLoS One 4:e4338.

Niedermeyer E, Lopes da Silva F (2005) Electroencephalography: basic principles, clinical applications, and related fields. Philadelphia: Lippincott Williams and Wilkins.

Ojemann G (2010) Cognitive mapping through electrophysiology. Epilepsia 51 [Suppl 1]:72-75.

Ojemann JG, Akbudak E, Snyder AZ, McKinstry RC, Raichle ME, Conturo TE (1997) Anatomic localization and quantitative analysis of gradient refocused echo-planar fMRI susceptibility artifacts. Neuroimage 6:156-167.

Peng CK, Buldyrev SV, Havlin S, Simons M, Stanley HE, Goldberger AL
(1994) Mosaic organization of DNA nucleotides. Phys Rev E Stat Phys Plasmas Fluids Relat Interdiscip Topics 49:1685-1689.

Pereira F, Mitchell T, Botvinick M (2009) Machine learning classifiers and fMRI: a tutorial overview. Neuroimage 45:S199-S209.

Posner MJ, Raichle ME (1997) Images of mind. New York: Freeman.

Poulet JF, Petersen CC (2008) Internal brain state regulates membrane potential synchrony in barrel cortex of behaving mice. Nature 454:881-885.

Raichle ME, MacLeod AM, Snyder AZ, Powers WJ, Gusnard DA, Shulman GL (2001) A default mode of brain function. Proc Natl Acad Sci U S A 98:676-682.

Rosen BR, Buckner RL, Dale AM (1998) Event-related functional MRI: past, present, and future. Proc Natl Acad Sci U S A 95:773-780.

Schroeder M (1991) Fractals, chaos, power laws: minutes from an infinite paradise. New York: Freeman.

Seeley WW, Menon V, Schatzberg AF, Keller J, Glover GH, Kenna H, Reiss AL, Greicius MD (2007) Dissociable intrinsic connectivity networks for salience processing and executive control. J Neurosci 27:2349-2356.

Shulman GL, Fiez JA, Corbetta M, Buckner RL, Miezin FM, Raichle ME, Petersen SE (1997) Common blood flow changes across visual tasks. II. Decreases in cerebral cortex. J Cogn Neurosci 9:648-663.

Sirotin YB, Das A (2009) Anticipatory haemodynamic signals in sensory cortex not predicted by local neuronal activity. Nature 457:475-479.

Talairach J, Tournoux P (1988) Co-planar stereotaxic atlas of the human brain. New York: Thieme Verlag.

Tolkunov D, Rubin D, Mujica-Parodi L (2010) Power spectrum scale invariance quantifies limbic dysregulation in trait anxious adults using fMRI: adapting methods optimized for characterizing autonomic dysregulation to neural dynamic time series. Neuroimage 50:72-80.

Vaishnavi SN, Vlassenko AG, Rundle MM, Snyder AZ, Mintun MA, Raichle ME (2010) Regional aerobic glycolysis in the human brain. Proc Natl Acad Sci U S A 107:17757-17762.

Vincent JL, Snyder AZ, Fox MD, Shannon BJ, Andrews JR, Raichle ME, Buckner RL (2006) Coherent spontaneous activity identifies a hippocampal-parietal mnemonic network. J Neurophysiol 96:35173531.

Vincent JL, Kahn I, Snyder AZ, Raichle ME, Buckner RL (2008) Evidence for a frontoparietal control system revealed by intrinsic functional connectivity. J Neurophysiol 100:3328-3342.

Voss RF, Clarke J (1975) "1/f noise" in music and speech. Nature 258:317-318.

Zarahn E, Aguirre GK, D’Esposito M (1997) Empirical analyses of BOLD fMRI statistics. I. Spatially unsmoothed data collected under nullhypothesis conditions. Neuroimage 5:179-197. 